\title{
ACTING WITH AWARENESS PREDICTS NEGATIVE BUT NOT POSITIVE AFFECT IN A SAMPLE OF ADDICTS
}

\author{
Tânia Caetano, Eduardo Ramadas, \& Jessica Lopes \\ Department or Research and Development, VillaRamadas International Treatment Centre (Portugal)
}

\begin{abstract}
Objective: Mindfulness has been associated with not only lower levels of psychopathology but also higher levels of positive psychological characteristics. Research has found that its impact is especially relevant in disorders related to emotion dysregulation, such as addiction. Mindfulness can be a valuable resource in addiction treatment and it is important to understand how the different dimensions may influence relevant therapeutic variables.

Our objective for this exploratory study was to not only explore the impact of the different mindfulness dimensions on symptoms of depressive, anxiety and stress, but also on the prevalence of positive and negative affect on the patients. Our hypothesis was that various dimensions of mindfulness would be significant predictors of both psychopathological symptoms, but also positive affect in treatment.

Method: The present study has a cross-sectional design, and was conducted as an exploratory analysis of the first results of a wider quasi-experimental study. Current results relate to 17 patients receiving treatment in VillaRamadas treatment center for addiction. The evaluation protocol was administrated before any mindfulness training was given. From the original and more extensive evaluation protocol, the relevant psychological measures for this study were the Kentucky Inventory of Mindfulness Skills (KIMS), the Depression, Anxiety and Stress Scale, 21 items (DASS-21), and the Positive Affect and Negative Affect Scale (PANAS).

Results: From 17 participants, 13 were male. Age varied from 19 to 64 years $(M=33, S D=10.13)$.

Age presented moderate negative associations with stress and negative affect. Years of education had a moderate positive association with the describing dimension of mindfulness. Acting with awareness presented strong negative associations with depression, anxiety and negative affect, and a very strong negative association with stress. Depression, anxiety and stress showed strong positive associations with negative affect, but only depression presented a strong negative association with positive affect.

The mindfulness dimension of acting with awareness, was a significant predictor of depression (35\%), anxiety $(42.9 \%)$, stress $(65.7 \%)$ and PANAS' negative affect (52.1\%). It was not a significant predictor of positive affect.

Discussion: The only dimension of mindfulness significantly associated with the measures of psychopathology and negative affect, was acting with awareness. Contrary to our original hypothesis, even this dimension did not present a significant association with positive affect. Being an exploratory study, more research needs to be conducted to validate and better understand these results.
\end{abstract}

Keywords: Mindfulness, acting with awareness, addiction, negative affect, positive affect.

\section{Introduction}

The interest in mindfulness has grown among psychology researchers and practitioners alike during the past two decades. Although a hard concept to describe due to its complex and rather foreigner cultural background, mindfulness or being mindful has been described as paying attention to the present moment in a purposeful and nonjudgmental way, with the goal of developing greater awareness, clarity and acceptance of the present reality (Kabat-Zinn, 1994).

Although some authors attempted to operationalize mindfulness as a single faceted construct, mainly through the development of unidimensional psychometric instruments (Buchheld, Grossman, \& Walach, 2001; Brown \& Ryan, 2003; Kumar, Feldman, \& Hayes, 2008), conceptualizing it as a both a multifaceted trait and set of different but related skills, allows for a more nuanced study of the construct and its relationship with other relevant psychological concepts such as affect. For the purpose of this study, a multifaceted operationalization was used with the Kentucky Inventory of Mindfulness Skills 
(KIMS; Baer, Smith, \& Allen, 2004), that measures four dimensions hat can also be conceptualized as skills to be trained. The considered dimensions are Observing (observing, noticing various stimuli such as internal or external phenomena), Describing (describing by applying words in a nonjudgmental way), Acting with awareness (being fully engaged in each activity), and Accepting without judgment (accept reality as it is without judging, avoiding or escaping it).

It has been an accepted reality that subjective well-being is not a unitary construct, but instead composed of two independent dimensions: positive affect and negative affect (Bradburn, 1969; Diener \& Emmons, 1985).

Positive affect has been shown to promote a range of psychological and physical health outcomes and some studies have found it more predictive of resilience, physical health and life satisfaction than the absence of negative affect (e.g. Cohen, Alper, Doyle, Treanor, \& Turner, 2006; Cohn, Fredrickson, Brown, Mikels, \& Conway, 2009). Because of their independence, the reduction of negative affect does not lead directly to the promotion of positive affect (Watson \& Clark, 1997), underlining the importance of understanding how different psychological constructs and subsequent therapies influence both the reduction of negative affect and the promotion of positive affect.

Mindfulness has been positively associated with various positive psychological constructs such as life satisfaction (Brown \& Ryan, 2003), self-esteem (Brown \& Ryan, 2003; Rasmussen \& Pidgeon, 2011) and positive affect (Brown \& Ryan, 2003), and negatively associated with negative affect such as depression (Cash \& Whittingham, 2010), neuroticism (Giluk, 2009) and general psychopathological symptoms (Baer et al., 2006).

However, the independence of positive and negative affect has been of some note in previous studies looking into the impact of mindfulness meditation and mindfulness-based interventions. A meta-analysis conducted by Sedlmeier et al. (2012), found that for nonclinical samples, meditation had particularly strong effect on negative affect. Another more recent study from Menezes and Bizarro (2015), a pilot study investigating the effect of brief training of focused meditation on a nonclinical sample, found significant changes regarding negative affect and trait-anxiety, but reported no change on positive affect.

Although it is difficult to generalize these few results, specially taking into account the different conceptualizations of mindfulness, it at least raises the question of how mindfulness impacts positive affect and negative affect differentially, especially taking into consideration non-clinical and clinical samples.

In this exploratory study, we proposed ourselves to study the relationship between the four dimensions of mindfulness suggested by Baer, Smith, \& Allen (2004), and both negative emotional states such as depression, anxiety and stress, and negative and positive affect, in a clinical sample of addicts.

\section{Methods}

\subsection{Participants and procedure}

The sample of the present exploratory study is a small sample composed of 17 Portuguese patients, all receiving treatment for an addiction disorder, at VillaRamadas International Treatment Centre throughout December of 2018 and January of 2019.

From the 17 patients, 13 were male and 4 were female, and age varied between 19 and 64 years $(M=33, S D=10.13)$. All the patients had been diagnosed at admission into treatment, either with a substance use disorder or with an impulsive disorder (behavioral addiction).

The relevant data for this study was collected as part of an experimental study looking at the impact of a 30-day program of mindfulness on top of treatment as usual in the treatment center. As part of this study, patients participated in a group session design to present the study and its goals and to allow informed consent, which was given in written from.

The evaluation protocol was administered by licensed clinical psychologists, members of the multidisciplinary staff.

\subsection{Measurements}

Kenucky Inventory of Mindfulness Skills (KIMS; Baer, Smith, \& Allen, 2004; Portuguese translation and adaptation by Carvalho, 2011), is a self-report instrument composed of 39 items scored on a 5-point Likert scale from 1 ("Never/Almost Never") to 5 ("Always"). Originally created to assess the efficacy of Dialectical Behavior Therapy, it measures the following four subscales: Observing (12 items), Describing (8 items), Acting with Awareness (10 items) and Accept without Judgement (9 items). Higher scores represent a greater report of the corresponding dimension. 
Depression, Anxiety and Stress Scale-21 (DASS-21; Lovibond \& Lovibond, 1995; Portuguese version by Pais-Ribeiro, Honrado, \& Leal, 2004), is a set of three self-report scales, each with 7 items scored on a 4-point Likert scale from 0 ("Did not apply to me at all") to 3 ("Applied to me very much or most of the time"), measuring depression, anxiety and stress. The total score of each scale can vary from 0 to 21 , with higher scores indicating higher levels of the emotional state. It is based on a dimensional conception of psychopathology, created on the assumption that differences in depression, anxiety and stress between normal and clinical population are differences of degree.

Positive Affect and Negative Affect Scale (PANAS; Watson \& Clark, 1988; Portuguese version by Galinha \& Pais-Ribeiro, 2005), is a set of two self-report scales, each one with 10 items scored on a 5-point Likert scale from 1 ("Not at all") to 5 ("Very much"), one measuring positive and another measuring negative affect. Each item consists of an emotion, positive or negative, and the participant was asked to consider the frequency with which he felt each emotion during the week prior. The total score of each scale can vary from 10 to 50, with higher scores indicating a greater report of the affect.

\subsection{Data analysis}

The IBM SPSS software package, version 23, was used to perform the necessary data analysis.

Descriptive statistics were used to explore the data and describe the sample.

Routine assumption testing was performed to better choose the appropriate statistical tests. Considering the small sample size, non-parametric alternatives were preferred.

Spearman correlation values we used as a non-parametric alternative to Pearson's correlation, with the intent of studying the relationships between the variables in study, more specifically, between the four dimensions of mindfulness considered in the present study and positive and negative affect.

Simple linear regression was used to test the possible predictive value of acting with awareness regarding the dependent variables.

\section{Results}

\subsection{Descriptive statistics}

Years of education ranged from 10 to 16 years, with a mean of 13.47 years $(S D=1.84)$.

The four mindfulness scales Observing, Describing, Acting with Awareness and Accept without Judgement, presented mean results of $37.50(S D=9.93), 27.87(S D=5.55), 32.13(S D=5.98), 26.44$ $(S D=4.95)$, respectively.

Depression showed a mean result of $6.82(S D=6.17)$, anxiety of $2.65(S D=2.87)$, and stress of $8.06(S D=5.70)$. $(S D=10.95)$.

Finally, positive affect had a mean result of $29.18(S D=11.49)$, and negative affect of 21.82

\subsection{Correlation values between the studied variables (Spearman's correlation)}

Age presented a moderate positive association with years of education, and moderate negative associations with stress and negative affect. Years of education had a moderate positive association with the describing dimension of mindfulness.

Acting with awareness was the only mindfulness dimension that showed significant correlation values with variables related to emotional states and affect, presenting strong negative associations with depression, anxiety and negative affect, and a very strong negative association with stress.

Depression, anxiety and stress showed strong positive associations with negative affect, but only depression presented a strong negative association with positive affect.

The complete results are presented in Table 1. 
Table 1. Correlation values between the studied variables.

\begin{tabular}{|c|c|c|c|c|c|c|c|c|c|c|c|}
\hline & 1 & 2 & 3 & 4 & 5 & 6 & 7 & 8 & 9 & 10 & 11 \\
\hline 1. Age & - & & & & & & & & & & \\
\hline $\begin{array}{l}\text { 2. Years of } \\
\text { Education } \\
\text { KIMS }\end{array}$ & $.510^{*}$ & - & & & & & & & & & \\
\hline 3. Observing & -.136 & .322 & - & & & & & & & & \\
\hline 4. Describing & .291 & $.539^{*}$ & $.499^{*}$ & - & & & & & & & \\
\hline $\begin{array}{l}\text { 5. Act with } \\
\text { Awareness }\end{array}$ & .475 & .099 & -.141 & .159 & - & & & & & & \\
\hline $\begin{array}{l}\text { 6. Accept } \\
\text { without }\end{array}$ & .064 & -.119 & .300 & -.194 & -.026 & - & & & & & \\
\hline $\begin{array}{l}\text { Judgement } \\
\text { DASS-21 }\end{array}$ & & & & & & & & & & & \\
\hline 7. Depression & -.264 & -.012 & -.251 & -.124 & $-.636^{* *}$ & -.403 & - & & & & \\
\hline 8. Anxiety & -.415 & -.057 & .237 & .091 & $-.681^{* *}$ & -.078 & $.744^{* *}$ & - & & & \\
\hline $\begin{array}{l}\text { 9. Stress } \\
\text { PANAS }\end{array}$ & $-.517^{*}$ & -.274 & .001 & -.170 & $-.834^{* *}$ & -.210 & $.852 * *$ & $.767^{* *}$ & - & & \\
\hline $\begin{array}{l}\text { 10. Positive } \\
\text { Affect }\end{array}$ & .291 & .123 & .445 & .321 & .223 & .470 & $-.616^{* *}$ & -.339 & -.438 & - & \\
\hline $\begin{array}{l}\text { 11. Negative } \\
\text { Affect }\end{array}$ & $-.589^{*}$ & -.215 & .071 & -.264 & $-.750^{* *}$ & -.042 & $.710^{* *}$ & $.764^{* * *}$ & $.788^{* *}$ & -.387 & - \\
\hline
\end{tabular}

\subsection{Simple linear regression}

Five simple linear regression analysis were conducted, to study the predictive value of the mindfulness subscale Acting with Awareness regarding the five dependent variables (DASS-2: depression, anxiety and stress; PANAS: positive affect and negative affect).

Acting with Awareness was a significant predictor of depression $(\mathrm{F}(1,14)=9.063, \mathrm{p}=.009)$, with an adjusted $R^{2}$ of .350 , anxiety $(\mathrm{F}(1,14)=12.275, \mathrm{p}=.004)$, with an adjusted $R^{2}$ of .429 , stress $(\mathrm{F}(1,14)=29.726, \mathrm{p}<.000)$, with an adjusted $R^{2}$ of .657 , and negative affect $(\mathrm{F}(1,14)=17.306, \mathrm{p}=.001)$, with an adjusted $R^{2}$ of .521 .

Lastly, Acting with Awareness was not a significant predictor of positive affect.

\section{Discussion}

The goal of the present study was to evaluate the possible differential impact of mindfulness, taking into consideration four dimensions, on negative affect (and more specifically depression, anxiety and stress) and positive affect in a clinical sample of addicts receiving treatment.

Interestingly, from the four dimensions of mindfulness considered (observing, describing, acting with awareness and accept without judgement), only acting with awareness presented moderate to strong significant associations with the relevant variables and only with those on the negative side of the emotional spectrum. Acting with awareness did not shown any association with positive affect in our sample. The only significant association with positive affect was a moderate negative association with depression, which was an expected result considering that lack of pleasure in previously pleasurable activities is one of the main criteria for a depression diagnosis.

From the theoretical conceptualization of each of the four dimensions of mindfulness considered, it would have been expected that instead of one of the frequently dubbed "what" dimensions (observing, describing and acting with awareness), the "how" dimension (accept without judgement) would have a particularly relevant role in emotion regulation and, therefore, the decrease of negative affect. In fact, studies looking into the impact of mindfulness in patients with auditory hallucinations of distressing voices (the perfect case study of high vulnerability to negative affect) found that acceptance significantly negatively associated with depression, anxiety, stress and negative affect (PANAS-negative; e.g. Brockman, Kiernan, \& Murrell, 2014).

Acting with awareness showed itself to be a significant predictor of depression, anxiety, stress and negative affect in general in our sample.

It is crucial that these results are interpreted taking into account the vast limitations of what is only an exploratory study. In addition to its cross-sectional design, that does not allow us to establish any causal relation, the sample size, which was very small, could be affecting the results, explaining the lack of association between the other mindfulness dimensions and the other studied variables. 
Nonetheless, the results raise interesting research questions that may be better explored in future studies with a more adequate sample and the possibility to consider the moderation value of other variables such as sex.

\section{References}

Baer, R. A., Smith, G. T., Allen, K. B. (2004). Assessment of mindfulness by self-report: the Kentucky inventory of mindfulness skills. Assessment, 11(3), 191-206.

Baer, R. A., Smith, G. T., Hopkins, J., Krietemeyer, J., \& Toney, L. (2006). Using self-report assessment methods to explore facets of mindfulness. Assessment, 13(1), 27-45.

Bradburn. N. M. (1969). The structure of psychological well-being. Chicago: Aldine.

Brockman, R., Kiernan, M., \& Murrell, E. (2014). Psychometric properties of two brief versions of the voices acceptance and action scale (VAAS): implications for the second-wave and third-wave behavioural and cognitive approaches to auditory hallucinations. Clin. Psychol. Psychother. doi: 10.1002/cpp.1916.

Brown, K. W., \& Ryan, R. M. (2003). The benefits of being present: Mindfulness and its role in psychological well.being. Journal of Personality and Social Psychology, 84, 822-848.

Buchheld, N., Grossman, P., \& Walach, H. (2001). Measuring mindfulness in insight meditation (Vipassana) and meditation based psychotherapy: The development of the Freiburg Mindfulness Inventory (FMI). Journal for Meditation and Meditative Research, 1, 11-34.

Carvalho, M.P.S. (2011). Validação dos Conceitos de Atenção Plena e Evitamento Experiencial segundo a Terapia da Aceitação e Compromisso (ACT) na Explicação do Comportamento Alimentar- Indicações para o Tratamento da Obesidade. Master's Degree Dissertation, Universidade Católica Portuguesa, Braga, Portugal.

Cash, M., \& Whittingham, K. (2010). What facets of mindfulness contribute to psychological well-being and depressive, anxious, and stress-related symptomatology? Mindfulness, 1, 177-182.

Cohen, S., Alper, C. M., Doyle, W. J., Treanor, J. J., \& Turner, R. B. (2006). Positive Emotional Style Predicts Resistance to Illness After Experimental Exposure to Rhinovirus or Influenza a Virus. Psychosomatic Medicine, 68(6), 809-815.

Cohn, M. A., Fredrickson, B. L., Brown, S. L., Mikels, J. A., \& Conway, A. M. (2009). Happiness Unpacked: Positive Emotions Increase Life Satisfaction by Building Resilience. Emotion, 9(3), 361-368.

Diener, E., \& Emmons, R. A. (1985). The Independence of Positive and Negative Affect. Journal of Personality and Social Psychology, 47(5), 1105-1117.

Galinha, I. O., \& Pais-Ribeiro, J. (2005). Contribuição para o estudo da versão portuguesa da Positive and Negative Affect Schedule (PANAS): II - Estudo psicométrico. Análise Psicológica, 2(23), 219-227.

Giluk, T. L. (2009). Mindfulness, big five personality, and affect: A meta-analysis. Personality and Individual Differences, 47, 805-811.

Kabat-Zinn, J. (1994). Wherever you go, there you are: mindfulness meditation in everyday life. New York: Hyperion.

Kumar, S. M., Feldman, G. C., \& Hayes, S. C. (2008). Changes in mindfulness end emotion regulation in an exposure based cognitive therapy dor depression. Cognitive Therapy \& Research, 32, 734-744.

Lovibond, S. H., \& Lovibond, P. F. (1995). Manual for the Depression Anxiety \& Stress Scales (second edition). Psychology Foundation.

Pais-Ribeiro, J., Honrado, A., \& Leal, I. (2004). Contribuição para o estudo da adaptação portuguesa das escalas de ansiedade, depressão e stress (eads) de 21 itens de lovibond e lovibond. Psicologia, Saúde \& Doenças, 5(2), 229-239.

Rasmussen, M. K., \& Pidgeon, A. M. (2011). The direct and indirect benefits of dispositional mindfulness on self-esteem and social anxiety. Anxiety, Stress \& Coping, 24, 227-233.

Sedlmeier, P., Eberth, J., Schwarz, M., Zimmermann, D., Haarig, F., Jaeger, S., \& Kunze, S. (2012). The psychological effects of meditation: A meta-analysis. Psychological Bulletin, 138(6), 1139-1171. doi: $10.1037 / \mathrm{a} 0028168$

Watson, D., \& Clark, L. A. (1988). Development and Validation of Brief Measures of Positive and Negative Affect: The PANAS Scales. Journal of Personality and Social Psychology, 54(6), 1063-1070.

Watson, D., \& Clark, L. A. (1997). Measurement and Mismeasurement of Mood: Recurrent and Emergent issues. Journal of Personality Assessment, 68(2), 267-296. 